\title{
Uniqueness and properties of positive solutions for infinite-point fractional differential equation with $p$-Laplacian and a parameter
}

\author{
Li Wang, Chengbo Zhai* \\ School of Mathematical Sciences, Shanxi University, Taiyuan 030006, Shanxi, P. R. China. \\ Communicated by D. Baleanu
}

\begin{abstract}
Using new methods for dealing with an infinite-point fractional differential equation with p-Laplacian and a parameter, we study the existence of unique positive solution for any given positive parameter $\lambda$, and then give some clear properties of positive solutions which depend on the parameter $\lambda>0$, that is, the positive solution $u_{\lambda}^{*}$ is continuous, strictly increasing in $\lambda$ and $\lim _{\lambda \rightarrow+\infty}\left\|u_{\lambda}^{*}\right\|=+\infty, \lim _{\lambda \rightarrow 0^{+}}\left\|u_{\lambda}^{*}\right\|=0$. Our analysis relies on some new theorems for operator equations $A(x, x)=x$ and $A(x, x)=\lambda x$, where $A$ is a mixed monotone operator. (C)2017 All rights reserved.
\end{abstract}

Keywords: Uniqueness, positive solution, p-Laplacian, infinite-point fractional differential equation, mixed monotone operator. 2010 MSC: 26A33, 34B18, 34B27.

\section{Introduction}

Recently, fractional calculus and fractional differential equations have been of great interest, and it is caused both by the intensive development of the theory of fractional calculus itself and by the applications of such constructions in various sciences such as mechanics, chemistry, and engineering. For details, see $[14,16,17]$ and references therein. For example, fractional differential equations have important applications in quantum mechanics, see $[7,12,13]$ for example. On the other hand, there are some papers reported on analytical and numerical methods for solving fractional differential equations, see $[8,14]$ for example.

In the paper [17], the authors considered the following infinite-point boundary value problem of fractional differential equations with p-Laplacian

$$
\begin{cases}D_{0+}^{\beta}\left(\varphi_{p}\left(D_{0+}^{\alpha} u(t)\right)\right)+f(t, u(t))=0, & 0<t<1, \\ u(0)=u^{\prime}(0)=\cdots=u^{(n-2)}(0)=0, & D_{0+}^{\alpha} u(0)=0, \quad u^{(i)}(1)=\sum_{j=1}^{\infty} \alpha_{j} u\left(\xi_{j}\right),\end{cases}
$$

and obtained the existence results of at least one positive solution. Their methods are upper and lower solutions, the Schauder fixed point theorem. But the uniqueness of positive solutions was not studied.

\footnotetext{
*Corresponding author

Email addresses: 985388806@qq. com (Li Wang), cbzhai@sxu.edu.cn (Chengbo Zhai)
} 
Moreover, we can find some results about infinite-point fractional differential equations in literature, see $[3-6,9,16,17]$ for example. But there are very few results on the uniqueness of positive solutions for infinite-point fractional boundary value problem. So we will discuss the following infinite-point fractional differential equation with $\mathrm{p}$-Laplacian and a parameter

$$
\begin{cases}D_{0+}^{\beta}\left(\varphi_{p}\left(D_{0+}^{\alpha} u(t)\right)\right)+\lambda f(t, u(t), u(t))=0, & 0<t<1, \\ u(0)=u^{\prime}(0)=\cdots=u^{(n-2)}(0)=0, & D_{0+}^{\alpha} u(0)=0, u^{(i)}(1)=\sum_{j=1}^{\infty} \alpha_{j} u\left(\xi_{j}\right),\end{cases}
$$

where $D_{0+}^{\alpha}, D_{0+}^{\beta}$ are the Riemann-Liouville derivatives, $\varphi_{p}(s)=|s|^{p-2} \cdot s, p>1, \lambda>0$ is a parameter, $f \in C([0,1] \times \mathbf{J}, \mathbf{J}), \mathbf{J}=[0,+\infty), i \in[1, n-2]$ is a fixed integer, $n-1<\alpha \leqslant n, n \geqslant 3,0<\beta \leqslant 1, \alpha_{j} \geqslant 0,0<$ $\xi_{1}<\xi_{2}<\cdots<\xi_{j-1}<\xi_{j}<\cdots<1(j=1,2, \cdots), \Delta-\sum_{j=1}^{\infty} \alpha_{j} \xi_{j}^{\alpha-1}>0$, here $\Delta=(\alpha-1)(\alpha-2) \cdots(\alpha-i)$.

As we know, fractional integral inequalities are useful in establishing the uniqueness of solutions for certain fractional differential equations, and they also provide upper and lower bounds for the solutions, see $[1,2]$ for example. Different from the works mentioned above, in this work, we will study the existence and uniqueness of positive solutions for the problem (1.1) for every parameter $\lambda>0$. Moreover, we give some clear properties of the positive solutions which depend on the parameter. Our methods are based upon some new results for operator equations $A(x, x)=x$ and $A(x, x)=\lambda x$.

\section{Preliminaries and several lemmas}

Here, we list some definitions and useful lemmas from fractional calculus theory.

Definition 2.1 ([11]). The Riemann-Liouville fractional integral of order $\alpha>0$ of a function $y:(0, \infty) \rightarrow \mathbf{R}$ is given by

$$
\mathrm{I}_{0+}^{\alpha} \mathrm{y}(\mathrm{t})=\frac{1}{\Gamma(\alpha)} \int_{0}^{\mathrm{t}}(\mathrm{t}-\mathrm{s})^{\alpha-1} \mathrm{y}(\mathrm{s}) \mathrm{ds}
$$

provided the right-hand side is pointwise defined on $(0, \infty)$.

Definition 2.2 ([11]). The Riemann-Liouville fractional derivative of order $\alpha>0$ of a continuous function $y:(0, \infty) \rightarrow \mathbf{R}$ is given by

$$
D_{0+}^{\alpha} y(t)=\frac{1}{\Gamma(n-\alpha)}\left(\frac{d}{d t}\right)^{n} \int_{0}^{t} \frac{y(s)}{(t-s)^{\alpha-n+1}} d s,
$$

where $n=[\alpha]+1,[\alpha]$ denotes the integer part of the number $\alpha$, provided that the right-hand side is pointwise defined on $(0, \infty)$.

Now, we consider the linear fractional differential equation with infinite-point boundary conditions

$$
\begin{cases}D_{0+}^{\alpha} u(t)+y(t)=0, & 0<t<1, \\ u(0)=u^{\prime}(0)=\cdots=u^{(n-2)}(0)=0, & u^{(i)}(1)=\sum_{j=1}^{\infty} \alpha_{j} u\left(\xi_{j}\right) .\end{cases}
$$

Lemma 2.3 ([17]). If $\mathrm{y} \in \mathrm{L}^{1}[0,1]$, then the unique solution of (2.1) can be written by

$$
u(t)=\int_{0}^{1} G(t, s) y(s) d s
$$

where

$$
G(t, s)=\frac{1}{p(0) \Gamma(\alpha)} \begin{cases}t^{\alpha-1} p(s)(1-s)^{\alpha-1-i}-p(0)(t-s)^{\alpha-1}, & 0 \leqslant s \leqslant t \leqslant 1, \\ t^{\alpha-1} p(s)(1-s)^{\alpha-1-i}, & 0 \leqslant t \leqslant s \leqslant 1,\end{cases}
$$

and $p(s)=\Delta-\sum_{s \leqslant \xi_{j}} \alpha_{j}\left(\frac{\xi_{j}-s}{1-s}\right)^{\alpha-1}(1-s)^{i}$. Obviously, $G(t, s)$ is continuous on $[0,1] \times[0,1]$. 
Lemma $2.4([10,17])$. The function $\mathrm{G}(\mathrm{t}, \mathrm{s})$ defined by $(2.2)$ has the following properties:

(1) $G(t, s) \geqslant \frac{1}{p(0) \Gamma(\alpha)} m_{1} s(1-s)^{\alpha-1-i} \cdot t^{\alpha-1}, \quad t, s \in[0,1]$;

(2) $G(t, s) \leqslant \frac{1}{p(0) \Gamma(\alpha)} p(s)(1-s)^{\alpha-1-i} \cdot t^{\alpha-1}, \quad t, s \in[0,1]$;

(3) $G(t, s)>0, \quad t, s \in(0,1)$,

where $\mathrm{m}_{1}=\inf _{0<s \leqslant 1} \frac{\mathrm{p}(\mathrm{s})-\mathrm{p}(0)}{\mathrm{s}}$ is a positive number.

Let $q>1$ satisfy $\frac{1}{p}+\frac{1}{q}=1$. Then $\varphi_{p}^{-1}(s)=\varphi_{q}(s)$. To discuss the problem (1.1), we first study the following linear boundary value problem

$$
\begin{cases}D_{0+}^{\beta}\left(\varphi_{p}\left(D_{0+}^{\alpha} u(t)\right)\right)+y(t)=0, & 0<t<1, \\ \mathfrak{u}(0)=u^{\prime}(0)=\cdots=u^{(n-2)}(0)=0, & D_{0+}^{\alpha} \mathfrak{u}(0)=0, u^{(i)}(1)=\sum_{j=1}^{\infty} \alpha_{j} u\left(\xi_{j}\right)\end{cases}
$$

for $y \in \mathrm{L}^{1}[0,1]$ with $y \geqslant 0$.

Lemma 2.5 ([17]). The linear problem (2.3) has the following form of solution

$$
u(t)=\left(\frac{1}{\Gamma(\beta)}\right)^{q-1} \int_{0}^{1} G(t, s) \varphi_{p}^{-1}\left(\int_{0}^{s}(s-\tau)^{\beta-1} y(\tau) d \tau\right) d s,
$$

where $\mathrm{G}(\mathrm{t}, \mathrm{s})$ is given by $(2.2)$.

\section{Main results}

Suppose that $(E,\|\cdot\|)$ is a real Banach space which is partially ordered by a cone $P \subset E$, by $\theta$ we denote the zero element of $E$. For $x, y \in E$, the notation $x \sim y$ means that there exist $k>0$ and $\mu>0$ such that $k x \leqslant y \leqslant \mu x$. Clearly, $\sim$ is an equivalence relation. Given $h>\theta$ (i.e., $h \geqslant \theta$ and $h \neq \theta$ ), we denote by $P_{h}$ the set $P_{h}=\{x \in E \mid x \sim h\}$. It is easy to see that $P_{h} \subset P$ is convex and $l P_{h}=P_{h}$ for all $l>0$.

Definition 3.1. $A: P \times P \rightarrow P$ is said to be a mixed monotone operator if $A(x, y)$ is increasing in $x$ and decreasing in $y$, i.e., $u_{i}, v_{i}(i=1,2) \in P, u_{1} \leqslant u_{2}, v_{1} \geqslant v_{2}$ implies $A\left(u_{1}, v_{1}\right) \leqslant A\left(u_{2}, v_{2}\right)$. Element $x \in P$ is called a fixed point of $A$ if $A(x, x)=x$.

In [15], Zhai and Zhang studied the following operator equations

$$
A(x, x)=x \text { and } A(x, x)=\lambda x,
$$

where $\mathrm{A}: \mathrm{P} \times \mathrm{P} \rightarrow \mathrm{P}$ is a mixed monotone operator and the following conditions are satisfied:

$\left(A_{1}\right)$ there exists $h \in P$ with $h \neq \theta$ such that $A(h, h) \in P_{h}$;

$\left(\mathrm{A}_{2}\right)$ for any $u, v \in P$ and $t \in(0,1)$, there exists $\varphi(t) \in(t, 1)$ such that

$$
A\left(t u, t^{-1} v\right) \geqslant \varphi(t) A(u, v) .
$$

They obtained the existence and uniqueness results for the above equations.

Lemma 3.2. Let $\mathrm{P}$ be a normal cone of $\mathrm{E}$, and $\left(\mathrm{A}_{1}\right)$ and $\left(\mathrm{A}_{2}\right)$ be satisfied. Then operator equation $\mathrm{A}(\mathrm{x}, \mathrm{x})=\mathrm{x}$ has a unique positive solution $x^{*}$ in $\mathrm{P}_{h}$. Moreover, for any initial $x_{0}, y_{0} \in \mathrm{P}_{h}$, the sequences

$$
x_{n}=A\left(x_{n-1}, y_{n-1}\right), \quad y_{n}=A\left(y_{n-1}, x_{n-1}\right), \quad n=1,2, \ldots,
$$

satisfy $\left\|\mathrm{x}_{\mathrm{n}}-\mathrm{x}^{*}\right\| \rightarrow 0$ and $\left\|\mathrm{y}_{\mathrm{n}}-\mathrm{x}^{*}\right\| \rightarrow 0$ as $\mathrm{n} \rightarrow \infty$.

Lemma 3.3. Let $\mathrm{P}$ be a normal cone of $\mathrm{E}$, and $\left(\mathrm{A}_{1}\right)$ and $\left(\mathrm{A}_{2}\right)$ be satisfied. Suppose that $\mathrm{x}_{\lambda}(\lambda>0)$ is the unique solution of parameter equation $A(x, x)=\lambda x$ in $P_{h}$. Then the following conclusions hold: 
$\left(\mathrm{B}_{1}\right)$ if $\varphi(\mathrm{t})>\mathrm{t}^{\frac{1}{2}}$ for $\mathrm{t} \in(0,1)$, then $\mathrm{x}_{\lambda}$ is strictly decreasing in $\lambda$, that is, $0<\lambda_{1}<\lambda_{2}$ implies $x_{\lambda_{1}}>x_{\lambda_{2}}$;

$\left(B_{2}\right)$ if there exists $\beta \in(0,1)$ such that $\varphi(t) \geqslant t^{\beta}$ for $t \in(0,1)$, then $x_{\lambda}$ is continuous in $\lambda$, that is, $\lambda \rightarrow \lambda_{0}\left(\lambda_{0}>0\right)$ implies $\left\|x_{\lambda}-x_{\lambda_{0}}\right\| \rightarrow 0$;

$\left(B_{3}\right)$ if there exists $\beta \in\left(0, \frac{1}{2}\right)$ such that $\varphi(t) \geqslant t^{\beta}$ for $t \in(0,1)$, then

$$
\lim _{\lambda \rightarrow+\infty}\left\|x_{\lambda}\right\|=0, \quad \lim _{\lambda \rightarrow 0^{+}}\left\|x_{\lambda}\right\|=+\infty .
$$

Our purpose of this section is to apply Lemmas 3.2 and 3.3 to study the problem (1.1), and obtain some new results on the existence and uniqueness of positive solutions. Moreover, we show that the positive solution with respect to $\lambda$ has some clear properties. It should be pointed out that the method used here is new to the literature and so are the existence and uniqueness results to the infinite-point fractional differential equations.

Next we will discuss the problem (1.1) in the Banach space $C[0,1]=\{x:[0,1] \rightarrow R$ is continuous $\}$. The norm of $E$ is $\|x\|=\sup \{|x(t)|: t \in[0,1]\}$. The cone of $E$ is given as $P=\{x \in C[0,1] \mid x(t) \geqslant 0, t \in[0,1]\}$, a standard, normal cone in $C[0,1]$. This space can be equipped with a partial order given by $x, y \in$ $C[0,1], x \leqslant y \Leftrightarrow x(t) \leqslant y(t)$ for $t \in[0,1]$.

We give the following conditions:

$\left(\mathrm{H}_{1}\right) \mathrm{f}(\mathrm{t}, \mathrm{x}, \mathrm{y}):[0,1] \times[0,+\infty) \times[0,+\infty) \rightarrow[0,+\infty)$ is a continuous function;

$\left(\mathrm{H}_{2}\right) \mathrm{f}(\mathrm{t}, \mathrm{x}, \mathrm{y})$ is increasing in $x$ for each $t \in[0,1]$ and $y \in[0,+\infty)$; and decreasing in $y$ for each $t \in[0,1]$ and $x \in[0,+\infty)$;

$\left(\mathrm{H}_{3}\right)$ for $r \in(0,1)$, there exists $\gamma \in(0, p-1)$ such that $f\left(t, r x, r^{-1} y\right) \geqslant r^{\gamma} f(t, x, y), t \in[0,1], x, y \in[0,+\infty)$;

$\left(\mathrm{H}_{4}\right) \mathrm{f}(\mathrm{t}, 0,1) \not \equiv 0$ for $\mathrm{t} \in[0,1]$.

Theorem 3.4. Assume that $\left(\mathrm{H}_{1}\right)-\left(\mathrm{H}_{4}\right)$ hold, then

(a) for every $\lambda \in(0,+\infty)$, the problem (1.1) has a unique positive solution $\mathrm{u}_{\lambda}^{*}$ in $\mathrm{P}_{\mathrm{h}}$, where $\mathrm{h}(\mathrm{t})=\mathrm{t}^{\alpha-1}, \mathrm{t} \in[0,1]$. Moreover, for any initial values $\mathrm{u}_{0}, v_{0} \in \mathrm{P}_{\mathrm{h}}$, the sequences

$$
\begin{aligned}
& u_{n+1}(t)=\left(\frac{\lambda}{\Gamma(\beta)}\right)^{q-1} \int_{0}^{1} G(t, s) \varphi_{p}^{-1}\left(\int_{0}^{s}(s-\tau)^{\beta-1} f\left(\tau, u_{n}(\tau), v_{n}(\tau)\right) d \tau\right) d s, \\
& v_{n+1}(t)=\left(\frac{\lambda}{\Gamma(\beta)}\right)^{q-1} \int_{0}^{1} G(t, s) \varphi_{p}^{-1}\left(\int_{0}^{s}(s-\tau)^{\beta-1} f\left(\tau, v_{n}(\tau), u_{n}(\tau)\right) d \tau\right) d s,
\end{aligned}
$$

where $\mathrm{n}=0,1,2, \cdots$, must satisfy $\mathrm{u}_{\mathrm{n}}(\mathrm{t}) \rightarrow \mathrm{u}_{\lambda}^{*}(\mathrm{t}), v_{\mathrm{n}}(\mathrm{t}) \rightarrow v_{\lambda}^{*}(\mathrm{t})$ as $\mathrm{n} \rightarrow \infty$;

(b) $u_{\lambda}^{*}$ is continuous in $\lambda$, that is, $\left\|u_{\lambda}^{*}-u_{\lambda_{0}}^{*}\right\| \rightarrow 0$ as $\lambda \rightarrow \lambda_{0}\left(\lambda_{0}>0\right)$.

Proof. To begin with, from Lemma 2.5, the problem (1.1) has an integral formulation given by

$$
u(t)=\left(\frac{\lambda}{\Gamma(\beta)}\right)^{q-1} \int_{0}^{1} G(t, s) \varphi_{p}^{-1}\left(\int_{0}^{s}(s-\tau)^{\beta-1} y(\tau) d \tau\right) d s,
$$

where $G(t, s)$ is given as in Lemma 2.3. For any $u, v \in P$, we define

$$
A(u, v)(t)=\left(\frac{1}{\Gamma(\beta)}\right)^{q-1} \int_{0}^{1} G(t, s) \varphi_{p}^{-1}\left(\int_{0}^{s}(s-\tau)^{\beta-1} f(\tau, u(\tau), v(\tau)) d \tau\right) d s
$$

For $x \geqslant 0$, we have $\varphi_{p}^{-1}(x) \geqslant 0$. Noting that $\left(\frac{1}{\Gamma(\beta)}\right)^{q-1}>0$ and $G(t, s) \geqslant 0$, it is easy to check that $A: P \times P \rightarrow P$. In the sequel, we check that $A$ satisfies all assumptions of Lemma 3.2 by several steps.

First step, we prove that $A$ is a mixed monotone operator. In fact, for $u_{i}, v_{i} \in P, i=1,2$ with $u_{1} \geqslant$ $u_{2}, v_{1} \leqslant v_{2}$, we know that $u_{1}(t) \geqslant u_{2}(t), v_{1}(t) \leqslant v_{2}(t), t \in[0,1]$ and by $\left(H_{1}\right),\left(H_{2}\right),\left(H_{4}\right)$, Lemma 2.3 , and the fact that $\varphi_{\mathrm{p}}^{-1}$ is monotone increasing,

$$
A\left(u_{1}, v_{1}\right)(t)=\left(\frac{1}{\Gamma(\beta)}\right)^{q-1} \int_{0}^{1} G(t, s) \varphi_{p}^{-1}\left(\int_{0}^{s}(s-\tau)^{\beta-1} f\left(\tau, u_{1}(\tau), v_{1}(\tau)\right) d \tau\right) d s
$$




$$
\geqslant\left(\frac{1}{\Gamma(\beta)}\right)^{q-1} \int_{0}^{1} G(t, s) \varphi_{p}^{-1}\left(\int_{0}^{s}(s-\tau)^{\beta-1} f\left(\tau, u_{2}(\tau), v_{2}(\tau)\right) d \tau\right) d s=A\left(u_{2}, v_{2}\right)(t)
$$

Second step, we show that $A$ satisfies the condition $\left(A_{2}\right)$. From $\left(H_{3}\right)$, for $u, v \in P, r \in(0,1)$, we obtain

$$
\begin{aligned}
A\left(r u, r^{-1} v\right)(t) & =\left(\frac{1}{\Gamma(\beta)}\right)^{q-1} \int_{0}^{1} G(t, s) \varphi_{p}^{-1}\left(\int_{0}^{s}(s-\tau)^{\beta-1} f\left(\tau, r u(\tau), r^{-1} v(\tau)\right) d \tau\right) d s \\
& \geqslant\left(\frac{r^{\gamma}}{\Gamma(\beta)}\right)^{q-1} \int_{0}^{1} G(t, s) \varphi_{p}^{-1}\left(\int_{0}^{s}(s-\tau)^{\beta-1} f(\tau, u(\tau), v(\tau)) d \tau\right) d s \\
& =\left(r^{\gamma}\right)^{q-1} A(u, v)(t), \quad t \in[0,1] .
\end{aligned}
$$

Let $\varphi(t)=t^{\gamma(q-1)}, t \in(0,1)$, then $0<\gamma(q-1)<1$ and thus $\varphi(t) \in(t, 1)$ for $t \in(0,1)$. Hence,

$$
\mathrm{A}\left(\mathrm{tu}, \mathrm{t}^{-1} v\right) \geqslant \varphi(\mathrm{t}) \mathrm{A}(\mathrm{u}, v), \quad \forall \mathrm{u}, v \in \mathrm{P}, \mathrm{t} \in(0,1) .
$$

So the condition $\left(A_{2}\right)$ is satisfied.

Third step, we show that $A(h, h) \in P_{h}$. On one hand, note that $h(t)=t^{\alpha-1}, t \in[0,1]$, it follows from $\left(\mathrm{H}_{1}\right)-\left(\mathrm{H}_{4}\right)$ and Lemma 2.4 that

$$
\begin{aligned}
A(h, h)(t) & =\left(\frac{1}{\Gamma(\beta)}\right)^{q-1} \int_{0}^{1} G(t, s) \varphi_{p}^{-1}\left(\int_{0}^{s}(s-\tau)^{\beta-1} f(\tau, h(\tau), h(\tau)) d \tau\right) d s \\
& \geqslant\left(\frac{1}{\Gamma(\beta)}\right)^{q-1} \cdot \frac{1}{p(0) \Gamma(\alpha)} \int_{0}^{1} t^{\alpha-1} m_{1} s(1-s)^{\alpha-1-i} \varphi_{p}^{-1}\left(\int_{0}^{s}(s-\tau)^{\beta-1} f(\tau, h(\tau), h(\tau)) d \tau\right) d s \\
& =\left(\frac{1}{\Gamma(\beta)}\right)^{q-1} \cdot \frac{1}{p(0) \Gamma(\alpha)} \int_{0}^{1} m_{1} s(1-s)^{\alpha-1-i} \varphi_{p}^{-1}\left(\int_{0}^{s}(s-\tau)^{\beta-1} f\left(\tau, \tau^{\alpha-1}, \tau^{\alpha-1}\right) d \tau\right) d s \cdot h(t) \\
& \geqslant\left(\frac{1}{\Gamma(\beta)}\right)^{q-1} \cdot \frac{1}{p(0) \Gamma(\alpha)} \int_{0}^{1} m_{1} s(1-s)^{\alpha-1-i} \varphi_{p}^{-1}\left(\int_{0}^{s}(s-\tau)^{\beta-1} f(\tau, 0,1) d \tau\right) d s \cdot h(t) .
\end{aligned}
$$

On the other hand, also from $\left(\mathrm{H}_{1}\right)-\left(\mathrm{H}_{4}\right)$ and Lemma 2.4, we have that

$$
\begin{aligned}
A(h, h)(t) & =\left(\frac{1}{\Gamma(\beta)}\right)^{q-1} \int_{0}^{1} G(t, s) \varphi_{p}^{-1}\left(\int_{0}^{s}(s-\tau)^{\beta-1} f(\tau, h(\tau), h(\tau)) d \tau\right) d s \\
& \leqslant\left(\frac{1}{\Gamma(\beta)}\right)^{q-1} \cdot \frac{1}{p(0) \Gamma(\alpha)} \int_{0}^{1} t^{\alpha-1} p(s)(1-s)^{\alpha-1-i} \varphi_{p}^{-1}\left(\int_{0}^{s}(s-\tau)^{\beta-1} f(\tau, h(\tau), h(\tau)) d \tau\right) d s \\
& =\left(\frac{1}{\Gamma(\beta)}\right)^{q-1} \cdot \frac{1}{p(0) \Gamma(\alpha)} \int_{0}^{1} p(s)(1-s)^{\alpha-1-i} \varphi_{p}^{-1}\left(\int_{0}^{s}(s-\tau)^{\beta-1} f\left(\tau, \tau^{\alpha-1}, \tau^{\alpha-1}\right) d \tau\right) d s \cdot h(t) \\
& \leqslant\left(\frac{1}{\Gamma(\beta)}\right)^{q-1} \cdot \frac{1}{p(0) \Gamma(\alpha)} \int_{0}^{1} p(s)(1-s)^{\alpha-1-i} \varphi_{p}^{-1}\left(\int_{0}^{s}(s-\tau)^{\beta-1} f(\tau, 1,0) d \tau\right) d s \cdot h(t) .
\end{aligned}
$$

Let

$$
\begin{aligned}
& l_{1}=\int_{0}^{1} m_{1} s(1-s)^{\alpha-1-i} \varphi_{p}^{-1}\left(\int_{0}^{s}(s-\tau)^{\beta-1} f(\tau, 0,1) d \tau\right) d s, \\
& l_{2}=\int_{0}^{1} p(s)(1-s)^{\alpha-1-i} \varphi_{p}^{-1}\left(\int_{0}^{s}(s-\tau)^{\beta-1} f(\tau, 1,0) d \tau\right) d s .
\end{aligned}
$$

Since $f$ is continuous, $f(t, 0,1) \not \equiv 0$, we have $\int_{0}^{s}(s-\tau)^{\beta-1} f(\tau, 0,1) d \tau \geqslant 0$ with

$$
\int_{0}^{s}(s-\tau)^{\beta-1} f(\tau, 0,1) d \tau \not \equiv 0
$$


So

$$
\varphi_{p}^{-1}\left(\int_{0}^{s}(s-\tau)^{\beta-1} f(\tau, 1,0) d \tau\right) d s \geqslant 0
$$

with

$$
\varphi_{\mathrm{p}}^{-1}\left(\int_{0}^{s}(s-\tau)^{\beta-1} f(\tau, 1,0) d \tau\right) d s \not \equiv 0, s \in[0,1] .
$$

Note that $m_{1} s \leqslant p(s)$, we can get $0<l_{1} \leqslant l_{2}$. Consequently,

$$
A(h, h)(t) \geqslant\left(\frac{1}{\Gamma(\beta)}\right)^{q-1} \cdot \frac{1}{p(0) \Gamma(\alpha)} \cdot l_{1} \cdot h(t), \quad A(h, h)(t) \leqslant\left(\frac{1}{\Gamma(\beta)}\right)^{q-1} \cdot \frac{1}{p(0) \Gamma(\alpha)} \cdot l_{2} \cdot h(t) .
$$

So we have

$$
\left(\frac{1}{\Gamma(\beta)}\right)^{q-1} \cdot \frac{l_{1}}{p(0) \Gamma(\alpha)} \cdot h \leqslant A(h, h) \leqslant\left(\frac{1}{\Gamma(\beta)}\right)^{q-1} \cdot \frac{l_{2}}{p(0) \Gamma(\alpha)} \cdot h .
$$

Hence $A(h, h) \in P_{h}$, and the condition $\left(A_{1}\right)$ is satisfied.

From Lemma 3.2, it is clear that there exists $u_{\lambda^{\prime}}^{*} \in P_{h}$ such that $A\left(u_{\lambda^{\prime}}^{*}, u_{\lambda^{\prime}}^{*}\right)=\lambda^{\prime} u_{\lambda^{\prime}}^{*}$. Note that $\varphi(t)=t^{\gamma(q-1)}$ with $0<\gamma(q-1)<1$, Lemma $3.3\left(B_{2}\right)$ implies that $u_{\lambda^{\prime}}^{*}$ is continuous in $\lambda^{\prime}$. Set $\lambda^{\prime}=\left(\frac{1}{\lambda}\right)^{q-1}$, $u_{\lambda^{\prime}}^{*}=u_{\lambda}^{*}$. Then $A\left(u_{\lambda^{\prime}}^{*} u_{\lambda}^{*}\right)=\left(\frac{1}{\lambda}\right)^{q-1} u_{\lambda}^{*}$. That is $u_{\lambda}^{*}=\lambda^{q-1} A\left(u_{\lambda^{\prime}}^{*} u_{\lambda}^{*}\right)$. So we can check that $u_{\lambda}^{*}$ is a unique positive solution of the problem (1.1) for any given $\lambda>0$. Moreover, $u_{\lambda}^{*}$ is continuous in $\lambda$, that is, $\left\|u_{\lambda}^{*}-u_{\lambda_{0}}^{*}\right\| \rightarrow 0$ as $\lambda \rightarrow \lambda_{0}\left(\lambda_{0}>0\right)$.

Finally, let $A_{\lambda}=\lambda^{q-1} A$, then $A_{\lambda}$ also satisfies all the conditions of Lemma 3.2. By Lemma 3.2, for any initial values $u_{0}, v_{0} \in P_{h}$, we construct two sequences $u_{n+1}=A_{\lambda}\left(u_{n}, v_{n}\right), v_{n+1}=A_{\lambda}\left(v_{n}, u_{n}\right), n=$ $0,1,2, \cdots$, then we obtain $u_{n} \rightarrow u_{\lambda}^{*}, v_{n} \rightarrow u_{\lambda}^{*}$ as $n \rightarrow \infty$. That is,

$$
\begin{aligned}
& u_{n+1}(t)=\left(\frac{\lambda}{\Gamma(\beta)}\right)^{q-1} \int_{0}^{1} G(t, s) \varphi_{p}^{-1}\left(\int_{0}^{s}(s-\tau)^{\beta-1} f\left(\tau, u_{n}(\tau), v_{n}(\tau)\right) d \tau\right) d s \rightarrow u_{\lambda}^{*}(t), \\
& v_{n+1}(t)=\left(\frac{\lambda}{\Gamma(\beta)}\right)^{q-1} \int_{0}^{1} G(t, s) \varphi_{p}^{-1}\left(\int_{0}^{s}(s-\tau)^{\beta-1} f\left(\tau, v_{n}(\tau), u_{n}(\tau)\right) d \tau\right) d s \rightarrow u_{\lambda}^{*}(t),
\end{aligned}
$$

as $n \rightarrow \infty$.

In the following, we need the other condition:

$\left(\mathrm{H}_{3}\right)^{\prime}$ for $r \in(0,1)$, there exists $\gamma \in\left(0, \frac{p-1}{2}\right)$ such that $f\left(t, r x, r^{-1} y\right) \geqslant r^{\gamma} f(t, x, y), t \in[0,1], x, y \in[0,+\infty)$.

Theorem 3.5. Assume that $\left(\mathrm{H}_{1}\right),\left(\mathrm{H}_{2}\right),\left(\mathrm{H}_{3}\right)^{\prime}$, and $\left(\mathrm{H}_{4}\right)$ hold, then

(a) for every $\lambda>0$, the problem (1.1) has a unique positive solution $\mathrm{u}_{\lambda}^{*}$ in $\mathrm{P}_{\mathrm{h}}$, where $\mathrm{h}(\mathrm{t})=\mathrm{t}^{\alpha-1}, \mathrm{t} \in[0,1]$. Moreover, for any initial values $\mathrm{u}_{0}, v_{0} \in \mathrm{P}_{\mathrm{h}}$, the sequences

$$
\begin{aligned}
& u_{n+1}(t)=\left(\frac{\lambda}{\Gamma(\beta)}\right)^{q-1} \int_{0}^{1} G(t, s) \varphi_{p}^{-1}\left(\int_{0}^{s}(s-\tau)^{\beta-1} f\left(\tau, u_{n}(\tau), v_{n}(\tau)\right) d \tau\right) d s, \\
& v_{n+1}(t)=\left(\frac{\lambda}{\Gamma(\beta)}\right)^{q-1} \int_{0}^{1} G(t, s) \varphi_{p}^{-1}\left(\int_{0}^{s}(s-\tau)^{\beta-1} f\left(\tau, v_{n}(\tau), u_{n}(\tau)\right) d \tau\right) d s,
\end{aligned}
$$

where $\mathrm{n}=0,1,2, \cdots$, must satisfy $\mathrm{u}_{\mathrm{n}}(\mathrm{t}) \rightarrow \mathrm{u}_{\lambda}^{*}(\mathrm{t}), v_{\mathrm{n}}(\mathrm{t}) \rightarrow v_{\lambda}^{*}(\mathrm{t})$ as $\mathrm{n} \rightarrow \infty$;

(b) $u_{\lambda}^{*}$ is strictly increasing in $\lambda$, that is, $u_{\lambda_{1}}^{*} \leqslant u_{\lambda_{2}}^{*}, u_{\lambda_{1}}^{*} \neq u_{\lambda_{2}}^{*}$ for $0<\lambda_{1}<\lambda_{2}$;

(c) $u_{\lambda}^{*}$ is continuous in $\lambda$, that is, $\left\|u_{\lambda}^{*}-u_{\lambda_{0}}^{*}\right\| \rightarrow 0$ as $\lambda \rightarrow \lambda_{0}\left(\lambda_{0}>0\right)$;

(d) $\lim _{\lambda \rightarrow+\infty}\left\|u_{\lambda}^{*}\right\|=+\infty, \quad \lim _{\lambda \rightarrow 0^{+}}\left\|u_{\lambda}^{*}\right\|=0$. 
Proof. We also consider the operator

$$
A(u, v)(t)=\left(\frac{1}{\Gamma(\beta)}\right)^{q-1} \int_{0}^{1} G(t, s) \varphi_{p}^{-1}\left(\int_{0}^{s}(s-\tau)^{\beta-1} f(\tau, u(\tau), v(\tau)) d \tau\right) d s
$$

Similar to the proof of Theorem 3.4, we know that $\mathrm{A}: \mathrm{P} \times \mathrm{P} \rightarrow \mathrm{P}$ is a mixed monotone operator and satisfies

(i) $A(h, h) \in P_{h}$, where $h(t)=t^{\alpha-1}, t \in[0,1]$;

(ii) $A(t x, t y) \geqslant \varphi(t) A(x, y), x, y \in P, t \in(0,1)$, where $\varphi(t)=t^{\gamma(q-1)}, t \in(0,1)$.

Let $\beta=\gamma(q-1)$, then from $\left(H_{3}\right)^{\prime}$, we have $0<\gamma(q-1)<\frac{1}{2}$ and thus $t^{\frac{1}{2}}<\varphi(t)=t^{\beta}<1$. Therefore, from Lemma 3.2, there exists $u_{\lambda^{\prime}}^{*} \in P_{h}$ such that $A\left(u_{\lambda^{\prime}}^{*} u_{\lambda^{\prime}}^{*}\right)=\lambda^{\prime} u_{\lambda^{\prime}}^{*}$. Set $\lambda^{\prime}=\left(\frac{1}{\lambda}\right)^{q-1}, u_{\lambda^{\prime}}^{*}=u_{\lambda^{*}}^{*}$. Then $A\left(u_{\lambda}^{*}, u_{\lambda}^{*}\right)=\left(\frac{1}{\lambda}\right)^{q-1} u_{\lambda}^{*}$. That is, $u_{\lambda}^{*}=\lambda^{q-1} A\left(u_{\lambda}^{*}, u_{\lambda}^{*}\right)$ and then $u_{\lambda}^{*}$ is a unique positive solution of the problem (1.1) for any given $\lambda>0$. Further, from Lemma 3.3, $u_{\lambda^{\prime}}^{*}$ is strictly decreasing, continuous in $\lambda$ and

$$
\lim _{\lambda^{\prime} \rightarrow+\infty}\left\|u_{\lambda^{\prime}}^{*}\right\|=0, \quad \lim _{\lambda^{\prime} \rightarrow 0^{+}}\left\|u_{\lambda^{\prime}}^{*}\right\|=+\infty .
$$

So we can obtain that: (i) $u_{\lambda}^{*}$ is strictly increasing in $\lambda$, that is, $u_{\lambda_{1}}^{*} \leqslant u_{\lambda_{2}}^{*}, u_{\lambda_{1}}^{*} \neq u_{\lambda_{2}}^{*}$ for $0<\lambda_{1}<\lambda_{2}$; (ii) $u_{\lambda}^{*}$ is continuous in $\lambda$, that is, $\left\|u_{\lambda}^{*}-u_{\lambda_{0}}^{*}\right\| \rightarrow 0$ as $\lambda \rightarrow \lambda_{0}\left(\lambda_{0}>0\right.$ ); (iii) $\lim _{\lambda \rightarrow+\infty}\left\|u_{\lambda}^{*}\right\|=+\infty, \lim _{\lambda \rightarrow 0^{+}}\left\|u_{\lambda}^{*}\right\|=$ 0 .

Corollary 3.6. Assume that $\left(\mathrm{H}_{1}\right)-\left(\mathrm{H}_{4}\right)$ hold, then following infinite-point fractional differential equation with $p$ Laplacian

$$
\begin{cases}D_{0+}^{\beta}\left(\varphi_{p}\left(D_{0+}^{\alpha} u(t)\right)\right)+f(t, u(t), u(t))=0, & 0<t<1, \\ u(0)=u^{\prime}(0)=\cdots=u^{(n-2)}(0)=0, & D_{0+}^{\alpha} u(0)=0, \quad u^{(i)}(1)=\sum_{j=1}^{\infty} \alpha_{j} u\left(\xi_{j}\right)\end{cases}
$$

has a unique positive solution $\mathrm{u}^{*}$ in $\mathrm{P}_{\mathrm{h}}$, where $\mathrm{h}(\mathrm{t})=\mathrm{t}^{\alpha-1}, \mathrm{t} \in[0,1]$. Moreover, for any initial values $\mathrm{u}_{0}, v_{0} \in \mathrm{P}_{\mathrm{h}}$, constructing successively the sequences

$$
\begin{aligned}
& u_{n+1}(t)=\left(\frac{1}{\Gamma(\beta)}\right)^{q-1} \int_{0}^{1} G(t, s) \varphi_{p}^{-1}\left(\int_{0}^{s}(s-\tau)^{\beta-1} f\left(\tau, u_{n}(\tau), v_{n}(\tau)\right) d \tau\right) d s, \\
& v_{n+1}(t)=\left(\frac{1}{\Gamma(\beta)}\right)^{q-1} \int_{0}^{1} G(t, s) \varphi_{p}^{-1}\left(\int_{0}^{s}(s-\tau)^{\beta-1} f\left(\tau, v_{n}(\tau), u_{n}(\tau)\right) d \tau\right) d s,
\end{aligned}
$$

where $\mathrm{n}=0,1,2, \cdots$, we obtain $\mathrm{u}_{\mathrm{n}}(\mathrm{t}) \rightarrow \mathrm{u}^{*}(\mathrm{t}), v_{\mathrm{n}}(\mathrm{t}) \rightarrow \mathrm{u}^{*}(\mathrm{t})$ as $\mathrm{n} \rightarrow \infty$.

Remark 3.7. In literature, we have not seen such results as Theorem 3.4, Theorem 3.5, and Corollary 3.6 on infinite-point fractional differential equations. The methods used in literature are not mixed monotone operator's method. So our method is different from ones in literature. Our main results can not only guarantee the existence of a unique positive solution for any parameter, but also help to make two iterative schemes for approximating it. In addition, the positive solution with respect to the parameter has some clear properties.

\section{An example}

We consider the following fractional boundary value problem with p-Laplacian and a parameter,

$$
\left\{\begin{array}{l}
D_{0^{+}}^{\frac{1}{2}}\left(\varphi_{3}\left(D_{0^{+}}^{\frac{5}{2}} u\right)\right)(t)+\lambda\left[\sqrt[3]{u(t)}+\frac{1}{\sqrt[4]{u(t)+1}}\right] t^{3}=0, \quad 0<t<1, \\
u(0)=u^{\prime}(0)=0, D_{0^{+}}^{\frac{5}{2}} \mathfrak{u}(0)=0, u^{\prime}(1)=\sum_{j=1}^{\infty} \frac{2}{j^{2}} \mathfrak{u}\left(\frac{1}{j}\right) .
\end{array}\right.
$$


For this example, we know that $n=3, i=1,2,<\alpha=\frac{5}{2} \leqslant 3, \beta=\frac{1}{2} \in(0,1], p=3, \alpha_{j}=\frac{2}{j^{2}}, \xi_{j}=\frac{1}{j}, f(t, x, y)=$ $\left(\sqrt[3]{x}+\frac{1}{\sqrt[4]{y+1}}\right) t^{3}$. After a simple computation, we get $\Delta=\frac{5}{2}, \sum_{j=1}^{\infty} \alpha_{j} \xi_{j}^{\alpha-1} \approx 2.109<\Delta$. Evidently, $f(t, x, y)$ satisfies $\left(\mathrm{H}_{1}\right)$ and $\left(\mathrm{H}_{2}\right)$. Let $\gamma=\frac{1}{3}$, then $\gamma \in\left(0, \frac{p-1}{2}\right)=(0,1)$. Further, for $r \in(0,1), x \geqslant 0, y \geqslant 0$, we have

$$
f\left(t, r x, r^{-1} y\right)=\left(\sqrt[3]{r x}+\frac{1}{\sqrt[4]{\frac{1}{r} y+1}}\right) t^{3} \geqslant\left(r^{\frac{1}{3}} \sqrt[3]{x}+\frac{r^{\frac{1}{4}}}{\sqrt[4]{y+1}}\right) t^{3} \geqslant r^{\frac{1}{3}}\left(\sqrt[3]{x}+\frac{1}{\sqrt[4]{1+y}}\right) t^{3}=r^{\gamma} f(t, x, y) .
$$

In addition,

$$
f(t, 0,1)=\frac{1}{\sqrt[4]{2}} t^{3} \not \equiv 0, t \in[0,1] .
$$

So all the conditions of Theorem 3.5 are satisfied. From Theorem 3.5, we can claim that:

(a) for $\lambda>0$, the problem (4.1) has a unique positive solution $u_{\lambda}^{*}$ in $P_{h}$, where $h(t)=t^{\frac{3}{2}}, t \in[0,1]$. Moreover, for any initial values $\mathfrak{u}_{0}, v_{0} \in \mathrm{P}_{\mathrm{h}}$, the sequences

$$
\begin{aligned}
& u_{n+1}(t)=\frac{\lambda^{\frac{1}{2}}}{\sqrt[4]{\pi}} \int_{0}^{1} G(t, s) \varphi_{3}^{-1}\left(\int_{0}^{s}(s-\tau)^{-\frac{1}{2}}\left[\sqrt[3]{u_{n}(\tau)}+\frac{1}{\sqrt[4]{v_{n}(\tau)+1}}\right] \tau^{3} d \tau\right) d s, \\
& v_{n+1}(t)=\frac{\lambda^{\frac{1}{2}}}{\sqrt[4]{\pi}} \int_{0}^{1} G(t, s) \varphi_{3}^{-1}\left(\int_{0}^{s}(s-\tau)^{-\frac{1}{2}}\left[\sqrt[3]{v_{n}(\tau)}+\frac{1}{\sqrt[4]{u_{n}(\tau)+1}}\right] \tau^{3} d \tau\right) d s,
\end{aligned}
$$

where $n=0,1,2, \cdots$, must satisfy $u_{n}(t) \rightarrow u_{\lambda}^{*}(t), v_{n}(t) \rightarrow v_{\lambda}^{*}(t)$ as $n \rightarrow \infty$;

(b) $u_{\lambda}^{*}$ is strictly increasing in $\lambda$, that is, $u_{\lambda_{1}}^{*} \leqslant u_{\lambda_{2}}^{*}, u_{\lambda_{1}}^{*} \neq u_{\lambda_{2}}^{*}$ for $0<\lambda_{1}<\lambda_{2}$;

(c) $u_{\lambda}^{*}$ is continuous in $\lambda$, that is, $\left\|u_{\lambda}^{*}-u_{\lambda_{0}}^{*}\right\| \rightarrow 0$ as $\lambda \rightarrow \lambda_{0}\left(\lambda_{0}>0\right)$;

(d) $\lim _{\lambda \rightarrow+\infty}\left\|u_{\lambda}^{*}\right\|=+\infty, \lim _{\lambda \rightarrow 0^{+}}\left\|u_{\lambda}^{*}\right\|=0$.

Remark 4.1. From the above example, it is easy to find many functions which satisfy the conditions of Theorem 3.4, Theorem 3.5, and Corollary 3.6.

\section{Conclusions}

In this paper, we discuss an infinite-point fractional differential equation with p-Laplacian and a parameter. We obtain the existence and uniqueness of positive solutions for any given positive parameter $\lambda$. Our methods are new theorems for operator equations $A(x, x)=x$ and $A(x, x)=\lambda x$, where $A$ is a mixed monotone operator. We give some good properties of positive solutions which depend on the parameter $\lambda>0$, that is, the positive solution $u_{\lambda}^{*}$ is continuous, strictly increasing in $\lambda$, and $\lim _{\lambda \rightarrow+\infty}\left\|u_{\lambda}^{*}\right\|=$ $+\infty, \lim _{\lambda \rightarrow 0^{+}}\left\|u_{\lambda}^{*}\right\|=0$. The methods used here are different from the literature and so the main results are new.

\section{Acknowledgment}

The research was supported by the Youth Science Foundation of China (11201272) and Shanxi Province Science Foundation (2015011005), 131 Talents Project of Shanxi Province (2015).

\section{References}

[1] D. Baleanu, S. D. Purohit, J. C. Prajapati, Integral inequalities involving generalized Erdélyi-Kober fractional integral operators, Open Math., 14 (2016), 89-99. 1

[2] D. Baleanu, S. D. Purohit, F. Uçar, On Grüss type integral inequality involving the Saigo's fractional integral operators, J. Comput. Anal. Appl., 19 (2015), 480-489. 1 
[3] H.-L. Gao, X.-L. Han, Existence of positive solutions for fractional differential equation with nonlocal boundary condition, Int. J. Differ. Equ., 2011 (2011), 10 pages. 1

[4] L.-M. Guo, L.-S. Liu, Y.-H. Wu, Existence of positive solutions for singular fractional differential equations with infinitepoint boundary conditions, Nonlinear Anal. Model. Control, 5 (2016), 635-650.

[5] L.-M. Guo, L.-S. Liu, Y.-H. Wu, Existence of positive solutions for singular higher-order fractional differential equations with infinite-point boundary conditions, Bound. Value Probl., 2016 (2016), 22 pages.

[6] $\mathrm{L}$. Hu, Existence of solutions to a coupled system of fractional differential equations with infinite-point boundary value conditions at resonance, Adv. Difference Equ., 2016 (2016), 13 pages. 1

[7] D. Kumar, S. D. Purohit, A. Secer, A. Atangana, On generalized fractional kinetic equations involving generalized Bessel function of the first kind, Math. Probl. Eng., 2015 (2015), 7 pages. 1

[8] D. Kumar, J. Singh, D. Baleanu, Numerical computation of a fractional model of differential-difference equation, J. Comput. Nonlinear Dyn., 11 (2016), 6 pages. 1

[9] B.-X. Li, S.-R. Sun, Y. Sun, Existence of solutions for fractional Langevin equation with infinite-point boundary conditions, J. Appl. Math. Comput., 53 (2017), 683-692. 1

[10] X. Y. Lu, X. Q. Zhang, L. Wang, Existence of positive solutions for a class of fractional differential equations with $\mathrm{m}-$ point boundary value conditions, (Chinese) J. Systems Sci. Math. Sci., 34 (2014), 218-230. 2.4

[11] I. Podlubny, Fractional differential equations, An introduction to fractional derivatives, fractional differential equations, to methods of their solution and some of their applications, Mathematics in Science and Engineering, Academic Press, Inc., San Diego, CA, (1999). 2.1, 2.2

[12] S. D. Purohit, Solution of fractional partial differential equations related to quantum mechanics, Adv. Appl. Math. Mech., 5 (2013), 639-651. 1

[13] S. D. Purohit, S. L. Kalla, On fractional partial differential equations related to quantum mechanics, J. Phys. A, 44 (2011), 8 pages. 1

[14] H. M. Srivastava, D. Kumar, J. Singh, An efficient analytical technique for fractional model of vibration equation, Appl. Math. Model., 45 (2017), 192-204. 1

[15] C.-B. Zhai, L.-L. Zhang, New fixed point theorems for mixed monotone operators and local existence-uniqueness of positive solutions for nonlinear boundary value problems, J. Math. Anal. Appl., 382 (2011), 594-614. 3

[16] X.-Q. Zhang, Positive solutions for a class of singular fractional differential equation with infinite-point boundary value conditions, Appl. Math. Lett., 39 (2015), 22-27. 1

[17] Q.-Y. Zhong, X.-Q Zhang, Positive solution for higher-order singular infinite-point fractional differential equation with p-Laplacian, Adv. Difference Equ., 2016 (2016), 11 pages. 1, 2.3, 2.4, 2.5 